\title{
CONTINUOUS INDEPENDENCE AND THE ILIEFF-SENDOV CONJECTURE
}

\author{
MICHAEL J. MILLER
}

(Communicated by Clifford J. Earle, Jr.)

\begin{abstract}
A maximal polynomial is a complex polynomial that has all of its roots in the unit disk, one fixed root, and all of its critical points as far as possible from a fixed point. In this paper we determine a lower bound for the number of roots and critical points of a maximal polynomial that must lie on specified circles.
\end{abstract}

\section{INTRODUCTION}

In 1962 Sendov conjectured that if a polynomial (with complex coefficients) has all its roots in the unit disk, then within one unit of each of its roots lies a root of its derivative. Numerous attempts to verify this conjecture have led to over 30 papers, but have met with limited success (for references, see [Ma2, B]).

We begin by defining $S(n, \beta)$ to be the set of complex polynomials of degree $n$ that have all their roots in the unit disk and at least one root at $\beta$ and by defining $|P|_{\alpha}$ to be the distance between $\alpha$ and the closest root of $P^{\prime}$. Written in this notation, Sendov's conjecture asserts that if $P \in S(n, \beta)$ then $|P|_{\beta} \leq 1$.

Define $P \in S(n, \beta)$ to be maximal with respect to $\alpha$ if for every $Q \in$ $S(n, \beta)$ we have $|P|_{\alpha} \geq|Q|_{\alpha}$. These maximal polynomials exist since $|P|_{\alpha}$ is a continuous function of the roots of $P$, which are contained in a compact set. Clearly, we may restrict our attention to maximal polynomials when investigating statements like Sendov's conjecture.

Define the critical circle to be the circle with center $\alpha$ and radius $|P|_{\alpha}$. In 1984, based on extensive computer searches for maximal polynomials, the author made

Conjecture 1. Let $P \in S(n, \beta)$ be maximal with respect to $\alpha$, let $s$ be the number of roots of $P$ (counting multiplicities) on the unit circle, and let $r$ be the number of roots of $P^{\prime}$ (counting multiplicities) on the critical circle. Then

(1) $r=n-1$, and

Received by the editors June 29, 1990.

1980 Mathematics Subject Classification (1985 Revision). Primary 30C15.

Key words and phrases. Ilieff, Sendov, maximal polynomial, continuous independence.

This paper was supported in part by a Le Moyne College Summer Research Stipend. A sketch of these results was presented to the American Mathematical Society in January 1989. 
(2) given that all roots of $P^{\prime}$ lie on a circle, $s$ is as large as possible.

The conclusions of this conjecture are surprisingly strong and in fact are sufficient to allow one to compute examples of maximal polynomials in a number of cases. Such computations have already resulted in counterexamples [Mi, §4] to the conjecture that if $P \in S(n, \beta)$ then $|P|_{\beta / 2} \leq 1-|\beta| / 2$ [GRR, S].

Substantial support for Conjecture 1 can be provided by showing that $2 r+s \geq$ $n+1$. In [Mi, §3] the author proved an analog of this result under a restricted definition of maximality and with the additional hypothesis that $P$ has no multiple critical points on the critical circle. Here we prove this result in full generality.

The idea behind the proof is quite simple-if a polynomial $P \in S(n, \beta)$ has relatively few roots on the unit circle and relatively few critical points on the critical circle, then these roots "ought" to be independent of each other. If this is true, then one "ought" to be able to move the roots of $P^{\prime}$ that are on the critical circle away from $\alpha$ while leaving $\beta$ and the roots of $P$ on the unit circle fixed. Thus, such a polynomial would not be maximal.

Indeed, since a monic polynomial of degree $n$ has $n$ independent coefficients, one might expect to be able to independently move up to a total of $n$ roots of that polynomial and its derivative. Unfortunately, this is not the case. A straightforward computation establishes that

$$
\int_{-5}^{5}\left(z^{2}+a z+b\right)\left(z^{3}-15 z\right) d z=0
$$

regardless of the values of $a$ and $b$. This implies that every polynomial of degree 6 that has 0 and $\pm \sqrt{15}$ as critical points and -5 as a root must also have 5 as a root. Thus for a polynomial of degree 6 , a total of only 4 roots of the polynomial and its derivative can be enough to fix another root of that polynomial.

\section{CONTINUOUS INDEPENDENCE}

We now establish a lower bound on the number of roots that can be independently moved. Define a derived root of $P$ to be a pair $(z, d)$ such that $z$ is a simple root of the $d$ th derivative $P^{(d)}$ of $P$. Note that a polynomial may have more than one distinct derived root with the same $z$ value; for example, $P(z)=z^{3}-z$ has derived roots $(0,0)$ and $(0,2)$.

Let $\left\{\left(z_{i}, d_{i}\right): 1 \leq i \leq m\right\}$ be a set containing some of the derived roots of $P$. Associated with each derived root $\left(z_{i}, d_{i}\right)$ in this set is a smallest nonnegative integer $c_{i}$ such that $P^{(k)}\left(z_{i}\right)=0$ for $c_{i} \leq k \leq d_{i}$. This produces a set of equations

$$
P^{(k)}\left(z_{i}\right)=0 \text { for } 1 \leq i \leq m \text { and } c_{i} \leq k \leq d_{i},
$$

which completely characterizes these derived roots and their multiplicities. This set of equations can be thought of as a linear system of equations in the coefficients of $P$. We will say that our set of derived roots is continuously independent if this linear system has a solution for the coefficients of $P$ as continuous functions of $z_{1}, \ldots, z_{m}$. Roughly speaking, a set of derived roots is continuously independent if changing the $z_{i}$ 's by small but arbitrary amounts (while maintaining their multiplicities) requires only small changes in the coefficients of $P$. 
Since the roots of $P$ and its derivatives are continuous in the coefficients of $P$ [Ma1, p. 3], this means that small but arbitrary changes in the continuously independent roots can be achieved with only small changes in the rest of the roots of $P$ and its derivatives.

A lower bound on the number of roots that are sure to be continuously independent is given by

Theorem 2. Suppose that $P$ is a polynomial of degree $n$ and that $\left\{\left(z_{i}, d_{i}\right)\right.$ : $1 \leq i \leq m\}$ is a set of derived roots of $P$. If $\sum_{i=1}^{m}\left(d_{i}+1\right) \leq n+1$, then the set of derived roots is continuously independent.

Proof. Let $P(z)=\sum_{t=0}^{n} a_{t} z^{t}$. Then system (1) becomes

$$
\sum_{t=0}^{n} a_{t}\left[\frac{t !}{(t-k) !} z_{i}^{t-k}\right]=0 \quad \text { for } 1 \leq i \leq m \text { and } c_{i} \leq k \leq d_{i} .
$$

We will prove shortly that the coefficient matrix of this system has full rank. Given this, we can choose a subset of $a_{0}, \ldots, a_{n}$ that has a nonsingular coefficient matrix and solve the linear system (2) for the $a_{i}$ 's in this subset (treating the $a_{i}$ 's not in the subset as constants, with values determined by the original polynomial $P$ ). In the resulting solution, each $a_{i}$ is continuous in $z_{1}, \ldots, z_{m}$, and so the set of derived roots is continuously independent.

To complete the proof, then, we need only show that the coefficient matrix of the system (2) has full rank. It will be convenient to assume that the $z_{i}$ 's are distinct. If not, say if $z_{1}=z_{2}$, then we may replace the rows $P^{(k)}\left(z_{i}\right)=0$ for $1 \leq i \leq 2$ and $c_{i} \leq k \leq d_{i}$ by the rows $P^{(k)}\left(z_{1}\right)=0$ for $\min \left\{c_{1}, c_{2}\right\} \leq k \leq$ $\max \left\{d_{1}, d_{2}\right\}$. (This will add some irrelevant rows to the coefficient matrix.) Repeat this process until the remaining $z_{i}$ 's are distinct. If the resulting matrix has full rank, then our original matrix (which is the resulting matrix with its irrelevant rows deleted) will also have full rank. Thus, we may assume without loss of generality that the $z_{i}$ 's are distinct.

We will show by induction that the submatrix consisting of the first $\sum_{i=1}^{m}\left(d_{i}+1\right)$ columns of this matrix has full rank. Set $d=\sum_{i=1}^{m} d_{i}$ and for $s \geq d$, choose $b_{0}, \ldots, b_{s}$ so that

$$
f(z)=z^{s-d} \prod_{r=1}^{m}\left(z-z_{r}\right)^{d_{r}}=\sum_{t=0}^{s} b_{t} z^{t} .
$$

Note that $b_{s}=1$. Now $z_{i}$ is a root of $f$ of multiplicity $d_{i}$, so for $1 \leq i \leq m$ and $c_{i} \leq k \leq d_{i}$,

$$
\begin{aligned}
f^{(k)}\left(z_{i}\right) & = \begin{cases}z_{i}^{s-d} k ! \prod_{i \neq r=1}^{m}\left(z_{i}-z_{r}\right)^{d_{r}} & \text { if } k=d_{i}, \\
0 & \text { if } k<d_{i}\end{cases} \\
& =\sum_{t=0}^{s} b_{t}\left[\frac{t !}{(t-k) !} z_{i}^{t-k}\right] .
\end{aligned}
$$

The sum in (3) represents the row-by-row result of adding multiples of columns 0 through $s-1$ to column $s$ in the coefficient matrix of system (2). (For convenience we will number the columns starting with 0 , to correspond with the variable subscripts.) Now, replacing column $s$ by the column with entries $f^{(k)}\left(z_{i}\right)$ (i.e., replacing a column by the sum of itself and a linear combination 
of other columns) will not change the rank of the matrix. Do this for $s$ from $d+m-1$ down to $d$. Consider the submatrix now formed by columns $d$ through $d+m-1$ and rows with $k=d_{i}$. Dividing each row by the (nonzero) common factor $k ! \prod_{i \neq r=1}^{m}\left(z_{i}-z_{r}\right)^{d_{r}}$ leaves us with a Vandermonde matrix in $z_{1}, \ldots, z_{m}$. As these are distinct, the submatrix is nonsingular [H, p. 204] hence of full rank.

Consider the submatrix now formed by columns $d$ through $d+m-1$ and rows with $k<d_{i}$. Every entry in this submatrix is 0 .

Finally, consider the submatrix formed by those rows with $k<d_{i}$. By induction (replacing each $d_{i}$ with $d_{i}-1$ ), the first $\sum_{i=1}^{m} d_{i}$ columns of this submatrix (i.e., columns 0 through $d-1$ ) have full rank.

Thus, we have partitioned our matrix as follows:

$$
\begin{array}{lc}
\text { columns } 0 \text { to } d-1 & \text { columns } d \text { to } d+m-1 \\
\text { rows with } k=d_{i} & \left(\begin{array}{cc}
\text { unknown } & \text { full rank } \\
\text { rows with } k<d_{i}
\end{array}\left(\begin{array}{c}
\text { full rank } \\
0
\end{array}\right)\right.
\end{array}
$$

Such a matrix is itself of full rank. Thus, our original matrix is also of full rank.

Note that Theorem 2 is the best result possible, in the sense that $n+1$ cannot be replaced by a larger integer. As an example, let $a \neq 0$ and let $z_{1}$ be a nonzero root of $P(z)=(z+a)^{n}-a^{n}$. Then $\left\{(-a, n-1),(0,0),\left(z_{1}, 0\right)\right\}$ is a set of derived roots with $\sum\left(d_{i}+1\right)=n+2$. Changing $a$ will clearly force a change in $z_{1}$, so this set is not continuously independent.

\section{MaXimal polynomials}

We are now able to prove

Theorem 3. Suppose that $P \in S(n, \beta)$ is maximal with respect to $\alpha$. Let $s$ be the number of roots of $P$ (counting multiplicities) on the unit circle and let $r$ be the number of roots of $P^{\prime}$ (counting multiplicities) on the critical circle. Then $2 r+s \geq n+1$.

Proof. Suppose instead that $2 r+s \leq n$. We will derive a contradiction.

Each root of $P$ with multiplicity $n_{i}$ corresponds to a derived root of $P$ with $d_{i}=n_{i}-1$, and each root of $P^{\prime}$ with multiplicity $m_{i}$ corresponds to a derived root of $P$ with $d_{i}=m_{i}$. Let $S$ be the set of derived roots of $P$ corresponding to the roots of $P$ on the unit circle, the roots of $P^{\prime}$ on the critical circle, and $\beta$. If $|\beta|<1$ then $\beta$ is a simple root of $P$ [Mi, proofs of 2.8 and 2.9]. If $|\beta|=1$ then $\beta$ has been included already among the roots of $P$ on the unit circle. In either case, for the derived roots in $S, \sum\left(d_{i}+1\right) \leq \sum n_{i}+\sum\left(m_{i}+1\right)+1 \leq$ $s+2 r+1 \leq n+1$, so the roots in $S$ are continuously independent.

While holding $\beta$ and those roots of $P$ on the unit circle fixed, move those roots of $P^{\prime}$ on the critical circle away from $\alpha$, with the movement small enough that no roots of $P$ inside the unit circle go out of it and no roots of $P^{\prime}$ outside the critical circle go into it. Denote the polynomial thus formed by $Q$, so $Q \in S(n, \beta)$ and $|Q|_{\alpha}>|P|_{\alpha}$.

This contradicts the maximality of $P$. 


\section{REFERENCES}

[B] J. Brown, On the Ilieff-Sendov conjecture, Pacific J. Math 135 (1988), 223-232.

[GRR] A. Goodman, Q. Rahman, and J. Ratti, On the zeros of a polynomial and its derivative, Proc. Amer. Math. Soc. 21 (1969), 273-274.

[H] F. Hohn, Introduction to linear algebra, Macmillan, New York, 1972.

[Ma1] M. Marden, Geometry of polynomials, 2nd ed., Amer. Math. Soc., Providence, RI, 1966.

[Ma2] _ Conjectures on the critical points of a polynomial, Amer. Math. Monthly 90 (1983), 267-276.

[Mi] M. Miller, Maximal polynomials and the Ilieff-Sendov conjecture, Trans. Amer. Math. Soc. 321 (1990), 285-303.

[S] G. Schmeisser, Bemerkungen zu einer Vermutung von Ilieff, Math. Z. 111 (1969), 121-125.

Department of Mathematics, Le Moyne College, Syracuse, New York 13214

E-mail address: millermj@lemoyne.bitnet 